\title{
Noctuídeos (Lepidoptera, Noctuidae) coletados em quatro Áreas Estaduais de Conservação do Rio Grande do Sul, Brasil
}

\author{
Alexandre Specht ${ }^{1}$, José Augusto Teston ${ }^{2}$, Rocco Alfredo Di Mare ${ }^{3} \&$ Elio Corseuil ${ }^{4}$
}

${ }^{1}$ Curso de Ciências-Biologia, Departamento de Ciências Exatas e da Natureza, Universidade de Caxias do Sul-UCS. Alameda João Dal Sasso 800, 95700-000 Bento Gonçalves-RS, Brasil; Programa de Pós-Graduação em Biotecnologia, Instituto de Biociências, UCS. Rua Francisco Getúlio Vargas 1130, 95070-560 Caxias do Sul-RS, Brasil. aspecht@ucs.br.

${ }^{2}$ Curso de Ciências Biológicas, Faculdade de Filosofia, Ciências e Letras, Universidade de Cruz Alta-UNICRUZ. Rua Andrade Neves 308, 98025-810 Cruz Alta-RS, Brasil. jateston@unicruz.edu.br

${ }^{3}$ Departamento de Biologia, Centro de Ciências Naturais e Exatas, Universidade Federal de Santa Maria-UFSM. Campus Universitário, Faixa de Camobi-Km 9, 97105-900 Santa Maria-RS, Brasil. ram13@terra.com.br

${ }^{4}$ Faculdade de Biociências, Pontifícia Universidade Católica do Rio Grande do Sul-PUCRS. Caixa Postal 1429, 90619-900 Porto Alegre-RS, Brasil. corseuil@pucrs.br

\begin{abstract}
Owlet moths (Lepidoptera, Noctuidae) collected in four protected areas located in Rio Grande do Sul State, Brazil. The objective of this work was to analyze the composition of the Noctuidae communities at Reserva Biológica de Ibirapuitã (Alegrete) and at Parque Estadual do Turvo (Derrubadas), Parque Estadual de Rondinha (Sarandi) and Parque Estadual do Espigão Alto (Barracão). Ten light traps were used to collect the insects from November/2000 to February/ 2001, sampling one night per area. The lepidopterous were identified at family and subfamily levels and, when it was possible, at genera and species levels. A total of 6,374 noctuids belonging to 249 taxa, distributed in 17 subfamilies were collected. The large number of species observed in all areas emphasize the importance of Conservation State Unity for the biodiversity maintenance.
\end{abstract}

KEYwORDS. Biodiversity; community structure; faunistic inventory; light trap.

Resumo. Noctuídeos (Lepidoptera, Noctuidae) coletados em quatro Áreas Estaduais de Conservação do Rio Grande do Sul, Brasil. O objetivo deste estudo foi analisar a composição das comunidades de Noctuidae na Reserva Biológica do Ibirapuitã (Alegrete) e nos Parques Estaduais do Turvo (Derrubadas), Rondinha (Sarandi) e Espigão Alto (Barracão). Dez armadilhas luminosas foram utilizadas para coletar os insetos, de novembro de 2000 a fevereiro de 2001, amostrandose uma noite por local. Os lepidópteros foram identificados aos níveis de família, subfamília e, quando viável, até espécie. Foram coletados 6.374 noctuídeos pertencentes a 249 táxons, distribuídos em 17 subfamílias. Os resultados indicam a existência de número maior de noctuídeos no Estado e a importância das Unidades Estaduais de Conservação para a manutenção da biodiversidade.

Palavras-Chave. Armadilha luminosa; biodiversidade; estrutura da comunidade; inventariamento de fauna.

Os lepidópteros constituem uma das ordens mais diversas de insetos e têm representantes de fácil captura, especialmente as mariposas, que são atraídas por fontes luminosas (Gardiner 1995). Vários modelos de aparelhos e fontes luminosas são empregados na coleta de mariposas sempre apresentando grande sucesso tanto em relação à quantidade quanto à variedade (Muirhead-Thomson 1991; Fry \& Waring 1996). Por outro lado, Marinoni \& Dutra (1996) ressaltam que existem várias limitações relacionadas ao seu emprego como método para obtenção de amostragens, especialmente no que se refere à seletividade e efeitos ambientais sobre as coletas.

A exemplo de vários estudos em outros países (Barlow \& Woiwod 1989; Holloway \& Barlow 1992; Lin 1995; Chey et al. 1997; Intachat et al. 1997; Woiwod 1997; Usher \& Keiller 1998; Kitching et al. 2000), também no Brasil os lepidópteros noturnos têm sido utilizados para indicar diversidade (Ferreira et al. 1995; Lübeck et al. 1995; Marinoni \& Dutra 1996; Marinoni et al. 1997; Camargo 1999; Specht 2001; Specht \& Corseuil 2002b; Teston \& Corseuil 2004).

A família Noctuidae destaca-se entre os principais grupos de mariposas pela grande diversidade, especialmente na Região Neotropical (Heppner 1991). Suas espécies relacionam-se com diversos ambientes e seus recursos, especialmente durante o período larval; alimentam-se de folhas, caules, brotos, raízes, flores, frutos, sementes, detritos ou outros insetos; algumas espécies que se alimentam de plantas cultivadas constituemse pragas de extrema importância econômica (Holloway et al. 1992). A grande diversidade, por vezes aliada a espécies com níveis populacionais elevados faz com que essas mariposas constituam a maior proporção dos lepidópteros amostrados com armadilhas luminosas (Gaston 1988; Barlow \& Woiwod 1989; Chey et al. 1997; Camargo 1999). Assim, o estudo deste grupo pode contribuir para o conhecimento da maior parte das características de um determinado ambiente.

O Rio Grande do Sul está inserido em dois grandes Biomas: Mata Atlântica e Campos Sulinos (Brasil 2000) porém, apresenta grande diversidade de ambientes com características físicas, climáticas, topográficas e fitogeográficas que motivaram o estabelecimento de onze zonas fisiográficas (Arend 1997). Em função desta diversidade de ambientes, o 
Estado do Rio Grande do Sul, através do Departamento de Florestas e Áreas Protegidas (DEFAP) da Secretaria Estadual do Meio Ambiente - SEMA, vem implementando planos de manejo para suas Unidades Estaduais de Conservação.

Este estudo objetivou contribuir com conhecimentos sobre os noctuídeos ocorrentes em quatro Unidades Estaduais de Conservação.

\section{MATERIALE MÉTODOS}

Os exemplares foram coletados nas Unidades de Conservação mantidas pelo DEFAP/SEMA, caracterizadas a seguir, com base nas descrições constantes em SEMA (2002).

Reserva Biológica do Ibirapuitã (RBI) - Localizada na região da Campanha, no oeste do Estado, às margens do rio Ibirapuitã, é a única área de proteção integral a preservar porções de campos nativos e mata ciliar. A área de campo caracteriza-se pelo domínio de espécies de gramíneas com presença esparsa de espinilhos [Acacia spp. - Mimosaceae] e aroeiras-pretas [Myracrodruon urundeuva Fr. All. - Anacardiaceae]. São ainda encontrados afloramentos de rocha onde se destacam cactáceas. Dentre as espécies da mata ciliar, estão o angicovermelho [Parapiptadenia rigida (Bentham) Brenan Mimosaceae], o camboim [Myrciaria tenella (Dc.)Berg.Myrtaceae], a embira [Daphnopsis racemosa Griseb. Thymelaeaceae] e a espinheira-santa [Maytenus ilicifolia Reiss - Celastraceae]. Situa-se no município de Alegrete, entre as coordenadas $29^{\circ} 54^{\prime}$ e $29^{\circ} 57^{\prime} \mathrm{S}$; $55^{\circ} 45^{\prime}$ e $55^{\circ} 48^{\prime} \mathrm{W}$, com área de 351,42 ha.

Parque Estadual do Turvo (PET) - Localizado na região do Planalto, às margens do rio Uruguai, é coberto pela Floresta do Alto Uruguai (Floresta Estacional Decidual), com árvores de até $30 \mathrm{~m}$ de altura, como o cedro [Cedrela fissilis Vellozo Meliaceae], a grápia [Apuleia leiocarpa Vog. Macbride Caesalpiniaceae], a canjerana [Cabralea canjerana (Vell.) Mart. - Meliaceae] e o louro [Laurus nobilis L. - Lauraceae]. Situase no município de Derrubadas, entre as coordenadas $27^{\circ} 00^{\prime} \mathrm{e}$ $27^{\circ} 20^{\prime} \mathrm{S} ; 53^{\circ} 40^{\prime}$ e $54^{\circ} 10^{\prime} \mathrm{W}$, com área de 17.491,40 ha.

Parque Estadual de Rondinha (PER) - Localizado no Planalto divide-se em Floresta de Araucária (Floresta Ombrófila Mista) e campos (savanas) com destaque para pequenos butiazais Butia spp. - Arecaceae] e jaboticabais [Myrcia cauliflora Berg - Myrtaceae]. Sua área é relativamente pequena e sofre forte pressão de uso agrícola. Situa-se no município de Sarandí, entre as coordenadas $27^{\circ} 45^{\prime} \mathrm{e} 27^{\circ} 58^{\prime} \mathrm{S}$; $52^{\circ} 45^{\prime}$ e $52^{\circ} 55^{\prime} \mathrm{W}$, com área de 1.000 ha.

Parque Estadual de Espigão Alto (PEE) - Localizado no Planalto, próximo ao rio Uruguai no vale do Arroio Marmeleiro, preserva o maior fragmento de floresta de araucária (Floresta Ombrófila Mista) e porções menores da Floresta do Alto Uruguai (Floresta Estacional Decidual). Localiza-se no município de Barracão, entre as coordenadas $27^{\circ} 30^{\prime}$ e $27^{\circ} 45^{\prime} \mathrm{S}$; $51^{\circ} 20^{\prime}$ e $51^{\circ} 40^{\prime} \mathrm{W}$, com área de $1.331,9$ ha.

As coletas foram efetuadas empregando armadilhas luminosas, modelo Pensilvânia (Frost 1957), distribuídas em pontos representativos dos vários tipos de vegetação ocorrentes em cada Reserva. As armadilhas providas de lâmpadas fluorescentes ultravioleta modelo F15 T12 LN, com comprimentos de onda variando de 290 a 450 nanômetros com pico ao redor de 340 nanômetros, utilizaram, como fonte de energia, baterias de 12 volts. Na porção inferior de cada aparelho, foi fixado um tronco de cone plástico, cujo maior diâmetro media $32 \mathrm{~cm}$ e o menor $16 \mathrm{~cm}$, ao qual acoplou-se um balde plástico contendo três litros de álcool etílico 96GL. Foram instaladas a cerca de 2,0 $\mathrm{m}$ do nível do solo e ficaram ligadas do ocaso até a manhã seguinte, aproximadamente 12 horas. As coletas foram realizadas nas noites de $23 / 24$ de novembro de 2000, na Reserva Biológica do Ibirapuitã; $24 / 25$ de janeiro de 2001, no Parque Estadual do Turvo; 26/27 de janeiro de 2001, no Parque Estadual de Rondinha e, 21/22 de fevereiro de 2001, no Parque Estadual do Espigão Alto. O esforço de coleta compreendeu a utilização de dez armadilhas durante apenas uma noite em cada local.

Em laboratório, os Noctuidae foram separados e registrados em uma listagem preliminar das espécies ocorrentes no Estado. Todos os exemplares que suscitaram dúvidas foram, após observação da presença da abertura timpânica entre o metatórax e o abdome, que evidencia pertencerem a Noctuoidea, separados para estudos subseqüentes. Os exemplares, cuja identificação não pôde ser prontamente realizada, foram preparados em alfinetes entomológicos e incluídos na listagem ao nível de gênero ou de subfamília. Além destes, exemplares representativos de cada espécie foram incorporados à coleção do Laboratório de Entomologia, Museu de Ciências e Tecnologia da Pontifícia Universidade Católica do Rio Grande do Sul (MCTP). A identificação foi realizada com base na coleção de referência formada por estudos de inventariamento faunístico regional do MCTP (Specht \& Corseuil 1996, 1998, 2001, 2002a), Coleção do Museu Entomológico Ceslau Biezanko da Faculdade de Agronomia Eliseu Maciel, Universidade Federal de Pelotas (UFPel) e obras de Hampson (1903-1913), Seitz (1919-1944) e Poole et al. (1993). Utilizou-se a classificação proposta por Lafontaine \& Poole (1991) para Plusinae, e Poole (1989) para as demais subfamílias.

De posse dos dados, organizou-se uma tabela de freqüência/abundância com os nomes listados em ordem alfabética, por subfamília, gênero e espécies. Nas espécies em que Poole (1989) coloca o respectivo gênero em dúvida, o mesmo foi colocado entre colchetes.

\section{RESULTADOS E DISCUSSÃO}

Foram coletados 6.374 noctuídeos pertencentes a 17 subfamílias, identificando-se 249 táxons, sendo 180 ao nível específico, 42 ao nível de genérico e 27 que ainda permanecem apenas ao nível de subfamília (Tabela I).

Com referência à importância destas áreas de preservação, destaca-se que, com esforço relativamente pequeno, coletouse $180(50,8 \%)$ das espécies de Noctuidae já registradas para o Estado (Specht \& Corseuil 1996, 1998, 2001, 2002a) (Tabela II).

$\mathrm{Na}$ Tabela III destaca-se o grande número de espécies exclusivas de cada localidade (117); observa-se que 65 
ocorreram simultaneamente em duas, 49 em três e, apenas, 18 em todas. Entre os noctuídeos capturados simultaneamente em três ou nos quatro locais destaca-se a predominância de espécies normalmente relacionadas com gramíneas ou culturas de verão (Silva et al. 1968); as demais, em sua maioria, já foram consideradas como constantes em outros trabalhos no Estado (Link 1977, Specht \& Corseuil 2002b).

Da mesma forma, observou-se grande variação no número de espécies por subfamília entre os locais (Tabela II), melhor evidenciado nas subfamílias com maior diversidade (Amphipyrinae, Catocalinae, Hadeninae, Noctuinae e Ophiderinae). Tais variações podem decorrer das diferentes épocas de coleta ou da utilização de plantas hospedeiras específicas de cada ambiente próprio de cada Unidade de Conservação. Assim, na Reserva Biológica do Ibirapuitã, a maior parte das espécies relaciona-se com plantas típicas de ambiente de Campo enquanto as dos Parques relacionam-se mais com vegetação de floresta, o que vem ao encontro com as ponderações de Kitching et al. (2000).

A Reserva Biológica do Ibirapuitã pertence a uma área Prioritária de Conservação da Biodiversidade dos Campos Sulinos, categorizada como de extrema importância biológica (Brasil 2000). Neste local, observou-se a predominância de espécies relacionadas com a vegetação de campo, muitas das quais são hóspedes das gramíneas nativas (Silva et al. 1968) que ocorrem na área. Representantes da maior parte destes noctuídeos são encontrados na coleção do Museu Entomológico Ceslau Biezanko (UFPel), capturados entre 1930 e 1970 e, atualmente, não são facilmente encontradas em regiões onde foram implantadas gramíneas exóticas, tanto para produção de forragem como de grãos. Com relação à importância dessa Reserva para a manutenção da biodiversidade dos ecossistemas de campos destaca-se que, apesar de ser o local com menor riqueza de espécies coletadas $(\mathrm{n}=83)$, foi a unidade de conservação que apresentou o maior percentual de espécies $(34,9 \%)$ de ocorrência exclusiva, com 29 espécies (Tabela III). Isto, provavelmente, por ser a única, deste estudo, a representar o Bioma Campos Sulinos (Brasil 2000) com suas características fisiográficas particulares. Algumas espécies consideradas raras ou, pelo menos, infreqüentes como Agrotis canities (Grote, 1902), Cydosia rimata Draudt, 1927 e Elaphria interstriata (Hampson, 1909) foram coletadas em números expressivos (Tabela I) o que pode indicar que esta reserva ainda mantem condições favoráveis à manutenção destas espécies típicas deste ambiente. Apenas $14(21,5 \%)$ espécies foram coletadas simultaneamente em cada uma das demais Unidades de Conservação: 3 com o Parque Estadual Florestal do Turvo, 7 com o Parque Estadual de Rondinha e 4 com o Parque Estadual de Espigão Alto (Tabela III). Este pequeno número de espécies compartilhadas com as outras Unidades de Conservação, evidencia o isolamento dessa Reserva como parte de um Bioma distinto. Entretanto, entre os noctuídeos coletados simultaneamente em três locais, representados principalmente por espécies polífagas ou relacionados com plantas cultivadas, observou-se uma proporção maior (44,9\%), com 22 das 49 espécies (Tabela III).
As subfamílias Noctuinae, Plusiinae e Sarrothripinae, mais relacionadas com ambientes de vegetação aberta, apresentaram o número de espécies relativamente alto; Amphipyrinae, Catocalinae e Ophiderinae, tipicamente mais diversas em ambientes de floresta, foram relativamente pobres em espécies (Tabela II), o que é condizente com o esperado para o local (Scoble, 1992).

Devido às demais unidades de conservação (Parque Estadual Florestal do Turvo, Parque Estadual de Rondinha e Parque Estadual do Espigão Alto) encontrarem-se inseridas no Bioma Mata Atlântica (Brasil 2000) compartilham diversas características fisiográficas em comum. Atribui-se a este fato a observação de que a maior parte $(78,5 \%)$ das espécies $(n=65)$ observadas simultaneamente entre dois locais $(\mathrm{n}=51)$ estiveram presentes nestes Parques. Da mesma forma, observou-se a maior proporção $(55,1 \%)$ das espécies que ocorreram simultaneamente em três locais $(\mathrm{n}=27 / 49)$ (Tabela III). Ainda com relação ao Bioma, na Tabela II, evidencia-se a maior riqueza de espécies, especialmente em Amphipyrinae, Catocalinae e Ophiderinae, coerente com observações constantes em Scoble (1992).

No Parque Estadual Florestal do Turvo, delimitado dentro de uma área prioritária para a conservação da biodiversidade da Mata Atlântica e considerado de extrema importância biológica (Brasil 2000) foram coletadas 134 espécies de Noctuidae. Destas, 38 ocorreram unicamente neste parque, 170 das 220 (77,3 \%) espécies coletadas no Bioma Mata Atlântica. (Tabela III). Compartilhou 13 espécies com o Parque Estadual de Rondinha, 24 com o do Espigão Alto e apenas 3 com a Reserva Biológica do Ibirapuitã. Quando consideradas as espécies ocorrentes em três locais compartilhou mais 38, especialmente com os demais parques do Bioma Mata Atlântica $(\mathrm{n}=27)$. Tais resultados indicam que este Parque, bem preservado (observação local), que dispõe de área consideravelmente grande (17.491,40 ha) no Rio Grande do Sul e que se prolonga pelo estado de Santa Catarina e na Argentina, apresenta boas condições para manter espécies típicas de seu ambiente, algumas muito raramente encontradas no Estado [Stauropides superba (Druce, 1894), Acanthodica lignaris Schaus, 1894, Acanthodica penicillium (C. Felder \& Rogenhofer, 1774), entre outras]. Ressalta-se a presença de somente três espécies pertencentes a Noctuinae, conhecendose bem apenas Anicla ignicans (Guenée, 1852) cujas plantas hospedeiras são gramíneas (Silva et al. 1968). A espécie mais abundante foi Anticarsia gemmatalis Hübner, 1818 com 185 indivíduos provavelmente oriundos das plantações de soja situadas no entorno do Parque; entretanto sobre a segunda espécie com maior abundância, Perigea xylophasioides Guenée, 1852, não se conhece o hábito alimentar.

No Parque Estadual de Rondinha, foram coletadas 114 espécies das quais 19 foram únicas. Estes dados indicam que, apesar do tamanho reduzido deste Parque (1.000 ha) e da forte pressão de uso agrícola no seu entorno, um grande número de espécies representativas do local ainda encontra ambiente favorável ao seu desenvolvimento e à manutenção de suas populações. Por outro lado, observou-se a maior proporção 
Tabela I - Número de noctuídeos capturados com armadilha luminosa em quatro Áreas Estaduais de Conservação do Rio Grande do Sul (RBI Reserva Biológica do Ibirapuitã, PET - Parque Estadual do Turvo, PER - Parque Estadual de Rondinha e PEE - Parque Estadual do Espigão Alto).

Subfamília e espécies \Área Estadual de Conservação

$\begin{array}{cc}\text { RBI } & \text { P } \\ 26 \\ 2 \\ \\ 10 \\ \\ \\ \\ 1 \\ 10\end{array}$

PET PER $\quad$ PEE

\section{Acontiinae}

1. Acontia ardoris (Hübner, [1827-1831])

2. Acontia morides Schaus, 1894

3. Acontia ruffinellii (Biezanko, 1959)

4. Acontia sp.

5. Cydosia rimata Draudt, 1927

6. Drobeta carneopicta (Hampson, 1910)

7. Drobeta $\mathrm{sp} 1$

8. Drobeta $\mathrm{sp} 2$

9. Lithacodia mella (Schaus, 1894)

10. Micrantha janeira (Schaus, 1904)

11. Trogotorna persecta Hampson, 1910

12. Acontiinae sp.

10

\section{Acronictinae}

23

14

13. Calymniodes conchylis (Guenée, 1852)

\section{Agaristinae}

14. Arpia janeira Schaus, 1896

15. [Aucula] magnifica (Schaus, 1904)

\section{Amphipyrinae}

16. Amolita irrorata Hampson, 1910

17. Antachara denterna (Guenée, 1852)

18. Antachara diminuta (Guenée, 1852)

19. Apamea alia (Guenée, 1852)

20. Argyrosticta aurifundens (Walker, 1858)

21. Argyrosticta decumana (C. Felder \& Rogenhofer, 1874)

22. Bryolymnia bicon (Druce, 1889)

23. Bryolymnia sp.

24. Callopistria fimbripes (Walker, 1858)

25. Callopistria floridensis (Guenée, 1852)

26. Condica albigera (Guenée, 1852)

27. Condica concisa (Walker, 1856)

28. Condica cupentia (Cramer, 1780)

29. Condica samula (Druce, 1898)

30. Condica selenosa (Guenée, 1852)

31. Condica stelligera (Guenée, 1852)

32. Condica sutor (Guenée, 1852)

33. Condica $\mathrm{sp} 1$

34. Condica $\mathrm{sp} 2$

35. Condica $\mathrm{sp} 3$

36. Cropia plumbicincta Hampson, 1908

37. Cropia sp.

38. Elaphria agrotina (Guenée, 1852)

39. Elaphria atrisigna (Hampson, 1909)

40. Elaphria deltoides (Möschler, 1880)

23

15

2

3

\section{1}

$3 \quad 1$

$11 \quad 30$

1218

$\begin{array}{ll} & 2 \\ 2 & 1 \\ 1 & \end{array}$

41. Elaphria ditrigona (Jones, 1908)

42. Elaphria interstriata (Hampson, 1909)

43. Elaphria jalapensis (Schaus, 1894)

44. Elaphria jonea (Schaus, 1906)

45. Elaphria marmorata (Schaus, 1894)

46. Elaphria subobliqua (Walker, 1858)

47. Elaphria subrubens (Guenée, 1852)

48. Elaphria villicosta (Walker, 1858)

49. Elaphria sp1

181
3

6

17

12

1

9
14

5

2

$33 \quad 2$

2

102

2

16

2

32

4

249

1

2

80

1

100

\begin{tabular}{cccc} 
& 3 & 1 & \\
& 5 & 5 & 6 \\
& 23 & 2 & 8 \\
& 2 & 6 & \\
& 1 & & 1 \\
& 3 & 30 & 46 \\
81 & 16 & 124 & 24 \\
\hline
\end{tabular}

1

1

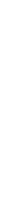

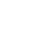

12

1
30
18
2
1
14
5
2
2
2
16


Tabela I - Continuação.

Subfamília e espécies \Área Estadual de Conservação

50. Elaphria $\mathrm{sp} 2$

51. Elaphria sp3

52. Elaphria $\mathrm{sp} 4$

53. Elaphria $\mathrm{sp} 5$

54. Elaphria sp6

55. Elaphria $\mathrm{sp} 7$

56. Elaphria $\mathrm{sp} 8$

57. Emarginea combusta (Walker, [1858])

58. Emarginea grammophora Guenée, 1852

59. Emarginea sp.

60. Galgula castra Schaus, 1898

61. Gonodes liquida (Möschler, 1886)

62. Hampsonodes bilineata (Maassen, 1890)

63. Hampsonodes infirma (Schaus, 1894)

64. Hampsonodes naevia (Guenée, 1852)

65. Hampsonodes xanthea (Jones, 1903)

66. Hampsonodes sp.

67. Heterochroma beryllus (Guenée, 1852)

68. Heterchroma sp 1

69. Heterochroma $\mathrm{sp} 2$

70. Macapta lurida (Schaus, 1898)

71. Macapta marginata (Schaus, 1904)

72. Macapta mursa (Schaus, 1894)

73. Magusa orbifera (Walker, 1857)

74. Perigea glaucoptera (Guenée, 1852)

75. Perigea secorva Schaus, 1906

76. Perigea xylophasioides Guenée, 1852

77. Perigea $\mathrm{sp} 1$

78. Perigea $\mathrm{sp} 2$

79. Phosphila lacruma (Schaus, 1894)

80. Phuphena fusipennis Walker, 1858

81. Phuphena petrovna (Schaus, 1894)

82. Phuphena transversa (Schaus, 1894)

83. Pseudina albina Hampson, 1910

84. Selambina trajiciens Walker 1858

85. [Sidemia] calidipes (Guenée, 1852)

86. Speocropia sp.

87. Spodoptera cosmioides (Walker, 1858)

88. Spodoptera dolichos (Fabricius, 1794)

89. Spodoptera eridania (Stoll, 1782)

90. Spodoptera frugiperda (J.E. Smith, 1797)

91. Spodoptera marima (Schaus, 1904)

92. Stauropides superba (Druce, 1894)

93. Trachea sp.

94. Aphipyrinae sp 1

95. Aphipyrinae sp2

96. Aphipyrinae sp3

97. Aphipyrinae sp4

98. Aphipyrinae sp5

99. Aphipyrinae sp6

Bagisarinae

100. Bagisara repanda (Fabricius, 1793)

Catocalinae

101. Acanthodica lignaris Schaus, 1894

\begin{tabular}{|c|c|c|c|}
\hline RBI & PET & PER & PEE \\
\hline & 5 & & 16 \\
\hline & 2 & 5 & 6 \\
\hline & 2 & 2 & 4 \\
\hline & 43 & 7 & 4 \\
\hline & & & 6 \\
\hline & 17 & & 4 \\
\hline & 2 & & 3 \\
\hline & 1 & & \\
\hline & 3 & & \\
\hline 3 & 8 & & 1 \\
\hline \multirow[t]{4}{*}{3} & & 1 & \\
\hline & 12 & 1 & \\
\hline & 7 & & 1 \\
\hline & 4 & 5 & 6 \\
\hline \multirow[t]{3}{*}{1} & 30 & 4 & 14 \\
\hline & 5 & 3 & 16 \\
\hline & 27 & 1 & 17 \\
\hline \multicolumn{4}{|l|}{3} \\
\hline \multirow{2}{*}{$\begin{array}{c}11 \\
1\end{array}$} & & 1 & 3 \\
\hline & & 24 & 8 \\
\hline \multirow[t]{10}{*}{8} & 1 & 1 & 1 \\
\hline & & & 4 \\
\hline & 8 & 6 & 170 \\
\hline & 147 & 9 & 7 \\
\hline & 1 & & 3 \\
\hline & & & 1 \\
\hline & 3 & & \\
\hline & & & 9 \\
\hline & & & 3 \\
\hline & & 7 & 4 \\
\hline \multirow[t]{3}{*}{1} & 2 & & 2 \\
\hline & & & 1 \\
\hline & 1 & & 1 \\
\hline \multicolumn{4}{|l|}{5} \\
\hline \multirow[t]{2}{*}{7} & 5 & 157 & 21 \\
\hline & & 1 & \\
\hline 1 & & 1 & 4 \\
\hline 4 & 1 & 12 & 5 \\
\hline \multirow[t]{2}{*}{12} & 2 & 10 & \\
\hline & 5 & & \\
\hline \multicolumn{4}{|l|}{1} \\
\hline & & & 1 \\
\hline & & & 2 \\
\hline & & & 1 \\
\hline & & & 1 \\
\hline & 2 & & \\
\hline & 1 & & 2 \\
\hline & 10 & 9 & 8 \\
\hline & 2 & & \\
\hline
\end{tabular}

Revista Brasileira de Entomologia 49(1): 130-140, março 2005 
Tabela I - Continuação.

\begin{tabular}{|c|c|c|c|c|}
\hline Subfamília e espécies $\backslash$ Área Estadual de Conservação & RBI & PET & PER & PEE \\
\hline 102. Acanthodica penicillium (C. Felder \& Rogenhofer, 1774) & & 4 & & \\
\hline 103. Acanthodica sp. & & 3 & & \\
\hline 104. Achaea ablunaris (Guenée, 1852) & & & 1 & \\
\hline 105. Herminocala pallidoides Poole, 1989 & & 7 & & \\
\hline 106. Mocis latipes Hübner, 1823 & & 3 & 6 & 9 \\
\hline 107. Mocis phasianoides (Guenée, 1852) & 2 & & & \\
\hline 108. Ophisma tecta Schaus, 1894 & & 1 & & \\
\hline 109. Ophisma tropicalis Guenée, 1852 & 1 & 43 & 69 & 2 \\
\hline 110. Ophisma variata Schaus, 1901 & & 6 & 50 & \\
\hline 111. Perasia helvina (Guenée, 1852) & & 1 & & \\
\hline 112. Ptichodes basilans (Guenée, 1852) & 3 & 1 & 1 & 3 \\
\hline 113. Zale exhausta (Guenée, 1852) & & 11 & 7 & 5 \\
\hline 114. Zale setipes (Guenée, 1852) & & & 1 & \\
\hline 115. Zale strigimacula (Guenée, 1852) & & 5 & & \\
\hline 116. Zale sp1 & & 93 & 11 & 3 \\
\hline 117. Zale sp2 & & 31 & 1 & 2 \\
\hline 118. Zale sp3 & & 5 & & \\
\hline \multicolumn{5}{|l|}{ Cuculliinae } \\
\hline 119. Cucullia argyrina Guenée, 1852 & 5 & & & 1 \\
\hline \multicolumn{5}{|l|}{ Euteliinae } \\
\hline 120. Paectes arcigera (Guenée, 1852) & 2 & & 3 & 3 \\
\hline 121. Paectes devincta (Walker, 1858) & & & 2 & 1 \\
\hline \multicolumn{5}{|l|}{ Hadeninae } \\
\hline 122. Boalda gyona (Schaus, 1929) & & & 9 & \\
\hline 123. Chabuata major (Guenée, 1852) & & & 1 & 2 \\
\hline 124. Chabuata sp1 & 7 & & & \\
\hline 125. Dargida meridionalis (Hampson, 1905) & 1 & & 2 & \\
\hline 126. Eriopyga approximans Jones, 1908 & 41 & & 95 & 22 \\
\hline 127. Eriopyga ditissima (Walker, 1857) & & 5 & 3 & 2 \\
\hline 128. Eriopyga punctulum Guenée, 1852 & & & & 4 \\
\hline 129. Eriopyga $\mathrm{sp} 1$ & & & & 8 \\
\hline 130. Eriopyga sp2 & & 57 & 3 & 49 \\
\hline 131. Faronta albilinea (Hübner, 1821) & 59 & 1 & 11 & \\
\hline 132. Hypotrix carneigera Guenée, 1852 & & 2 & & \\
\hline 133. Hypotrix flavigera Guenée, 1852 & & & 1 & 9 \\
\hline 134. Hypotrix proxima (Draudt, 1924) & & 41 & & \\
\hline 135. Hypotrix purpurigera Guenée, 1852 & & 2 & & 5 \\
\hline 136. Hypotrix sedecens (Schaus, 1903) & & 39 & & 3 \\
\hline 137. Hypotrix sp1 & & & 1 & \\
\hline 138. Hypotrix $\mathrm{sp} 2$ & & 5 & & 1 \\
\hline 139. Leucania albifasciata (Hampson, 1905) & & & 1 & 2 \\
\hline 140. Leucania cinereicolis Walker, 1858 & 15 & & 13 & 7 \\
\hline 141. Leucania humidicola Guenée, 1852 & 9 & 4 & 48 & 24 \\
\hline 142. Leucania latiuscula Herrich-Schäffer, 1868 & & 1 & & 10 \\
\hline 143. Leucania microsticha (Hampson, 1905) & 3 & & 17 & 1 \\
\hline 144. Leucania polystrota (Hampson, 1905) & & 1 & & \\
\hline 145. Leucania rivorum Guenée, 1852 & 3 & 7 & & 2 \\
\hline 146. Leucania steniptera (Hampson, 1905) & 7 & 1 & 3 & 1 \\
\hline 147. Leucania $\mathrm{sp} 1$ & & 1 & & 6 \\
\hline 148. Leucania $\mathrm{sp} 2$ & & & & 1 \\
\hline 149. Orthodes curvirena (Guenée, 1852) & 2 & 5 & 22 & 10 \\
\hline 150. Orthodes infirma Guenée, 1852 & 1 & 2 & 19 & 26 \\
\hline 151. [Polia] subjecta (Walker, 1857) & & & & 1 \\
\hline 152. Proteinaina achatioides (Guenée, 1852) & & & & 14 \\
\hline
\end{tabular}


Tabela I - Continuação.

Subfamília e espécies \Área Estadual de Conservação

$\begin{array}{lccc}\text { RBI } & \text { PET } & \text { PER } & \text { PEE } \\ 4 & & 20 & 24 \\ 2 & & & \\ 1 & & 1 & \end{array}$

\section{Heliothinae}

157. Helicoverpa gelotopoeon (Dyar, 1921)

2

158. Helicoverpa zea (Boddie, 1850)

159. Heliothis molochitina (Berg, 1882)

160. Heliothis parana Poole \& Mitter, 1993

161. Heliothis tergemina (C. Felder \& Rogenhofer, 1874)

162. Heliothis virescens (Fabricius, 1777)

163. Heliothinae sp 1

164. Heliothinae sp2

\section{Herminiinae}

165. Bleptina confusalis Guenée, 1852

166. Herminiinae sp1

167. Herminiinae sp2

168. Herminiinae sp3

Noctuinae

169. Agrotis brachystria (Hampson, 1903)

170. Agrotis canities (Grote, 1902)

171. Agrotis ipsilon (Hufnagel, 1766)

172. Agrotis subterranea (Fabricius, 1794)

173. Anicla ignicans (Guenée, 1852)

174. Anicla infecta (Ochsenheimer, 1816)

175. Anicla temperata (Schaus, 1894)

176. Anicla $\mathrm{sp} 1$

177. Ochropleura cirphisioides Köhler, 1955

178. Peridroma saucia (Hübner, 1808)

179. Pseudoleucania butleri (Schaus, 1898)

180. Pseudoleucania ochreicosta (Zerny, 1916)

181. Pseudoleucania tandiliense (Köhler, 1959)

182. Pseudoleucania sp1

183. Pseudoleucania sp2

184. Tandilia microstigma (Hampson, 1903)

185. Tandilia rodea (Schaus, 1894)

186. Tandilia sp1

187. Tripseuxoa deeringi Schaus, 1929

188. Tripseuxoa strigata Hampson, 1903

189. Noctuinae sp.

$$
1
$$

\section{Nolinae}

190. Nolinae sp.

\section{Ophiderinae}

191. Alabama argillacea (Hübner, 1823)

192. Anoba pohly (C. Felder \& Rogenhofer, 1874)

193. Anomis editrix (Guenée, 1852)

194. Anomis erosa Hübner, 1816

195. Anticarsia gemmatalis Hübner, 1818

196. Ascalapha odorata (Linnaeus, 1758)

22

\begin{tabular}{|c|c|c|c|}
\hline & & 1 & 4 \\
\hline & 33 & 1 & 6 \\
\hline & 2 & & \\
\hline & 1 & & 1 \\
\hline 22 & 185 & 1191 & 109 \\
\hline 1 & 1 & & \\
\hline & & 2 & 7 \\
\hline & & 2 & 20 \\
\hline & 13 & & \\
\hline & 5 & & \\
\hline & 18 & & \\
\hline
\end{tabular}


Tabela I - Continuação.

\begin{tabular}{|c|c|c|c|c|}
\hline Subfamília e espécies $\backslash$ Área Estadual de Conservação & RBI & PET & PER & PEE \\
\hline 202. Coenipeta bibitrix (Hübner, 1823) & & 1 & 2 & \\
\hline 203. Coenipeta zenobina Massen, 1890) & & 13 & 3 & \\
\hline 204. Cyclopis caecutiens Hübner, 1821 & & & 1 & \\
\hline 205. Encruphion leena (Druce, 1898) & & & & 3 \\
\hline 206. Epitausa flagrans (Walker, 1869) & & 16 & & \\
\hline 207. Euclystis guerini (Guenée, 1852) & 1 & 1 & & \\
\hline 208. Eulepidotis detracta (Walker, 1858) & & 4 & 3 & \\
\hline 209. Eulepidotis hemileuca (Guenée, 1852) & & 1 & & \\
\hline 210. Gabara grisea (Schaus, 1894) & 3 & 3 & & \\
\hline 211. Goniohelia gallinago (C. Felder \& Rogenhofer, 1874) & & 16 & 1 & \\
\hline 212. Gonodonta biarmata Guenée, 1852 & & 14 & & \\
\hline 213. Gonodonta bidens Geyer, 1832 & & & 2 & 3 \\
\hline 214. Hemicephalis agenoria (Druce, 1890) & & 1 & & \\
\hline 215. Herminodes carbonelli Biezanko, 1959 & 1 & 13 & & 2 \\
\hline 216. Herminodes renicula (C. Felder \& Rogenhofer, 1874) & & & 2 & \\
\hline 217. Hypocala andremona $($ Stoll, 1781$)$ & & & 2 & \\
\hline 218. Letis mineis Geyer, 1827 & & 7 & & \\
\hline 219. Licha undilinealis Walker, 1850 & & & & 1 \\
\hline 220. Makapta argentescens Schaus, 1904 & 1 & 22 & 1 & 3 \\
\hline 221. Melipotis cellaris (Guenée, 1852) & 5 & & & \\
\hline 222. Melipotis fasciolaris (Hübner, 1831) & 2 & & & \\
\hline 223. Melipotis perpendicularis (Guenée, 1852) & & 8 & 5 & 2 \\
\hline 224. Melipotis sp. & & & & 1 \\
\hline 225. Peteroma latizonata Hampson, 1926 & & & 1 & \\
\hline 226. Phrodita fasciata Jones, 1908 & 3 & & & 2 \\
\hline 227. Rhosologia tripuncta Schaus, 1901 & & 1 & 1 & \\
\hline 228. Selenisa sueroides (Guenée, 1852) & & 5 & & \\
\hline 229. Tautobriga euspila Walker, 1869 & & 2 & & \\
\hline 230. Ophiderinae sp1 & & 10 & & \\
\hline 231. Ophiderinae sp2 & & 30 & & \\
\hline 232. Ophiderinae sp3 & & 10 & & 1 \\
\hline 233. Ophiderinae sp4 & & 5 & 1 & \\
\hline 234. Ophiderinae sp5 & & 9 & & \\
\hline 235. Ophiderinae sp6 & & 2 & & \\
\hline 236. Ophiderinae sp7 & & & 1 & \\
\hline 237. Ophiderinae sp8 & & & 1 & \\
\hline \multicolumn{5}{|l|}{ Pantheinae } \\
\hline 238. Bathyra chavannesi (Felder and Rogenhofer, 1874) & & & & 3 \\
\hline \multicolumn{5}{|l|}{ Plusiinae } \\
\hline 239. Autographa bonaerensis (Berg, 1882) & 6 & & & \\
\hline 240. Autoplusia phytolacca (Sepp, [1848]) & & 1 & & \\
\hline 241. Ctenoplusia oxygramma (Geyer, 1832$)$ & 1 & & 2 & 4 \\
\hline 242. Mouralia tinctoides (Guenée, 1852) & 1 & & & \\
\hline 243. Pseudoplusia includens (Walker [1858]) & & & 13 & 2 \\
\hline 244. Rachiplusia nu (Guenée, 1852) & 24 & 3 & 25 & \\
\hline \multicolumn{5}{|l|}{ Sarrothripinae } \\
\hline 245. Iscadia aperta Walker, 1857 & 1 & & & \\
\hline 246. Iscadia canalalis (Schaus, 1938) & & & & 16 \\
\hline 247. Sarrothripinae sp1 & 2 & & 2 & 3 \\
\hline 248. Sarrothripinae sp2 & 16 & & & \\
\hline 249. Sarrothripinae sp3 & 2 & & & \\
\hline Total de indivíduos & 944 & 1482 & 2711 & 1237 \\
\hline
\end{tabular}


Tabela II - Número de espécies por subfamílias de noctuídeos coletados com armadilhas luminosas em Unidades de Conservação do Rio Grande do Sul: Reserva Biológica do Ibirapuitã (RBI), Parque Estadual do Turvo (PET), Parque Estadual de Rondinha (PER), Parque Estadual do Espigão Alto (PEE). Totais de noctuídeos identificados aos níveis específico (sp), de gênero ou de subfamília, distintos de todas as espécies referidas para o Rio Grande do Sul (nc). Número total de noctuídeos já registrado para o Rio Grande do Sul (Total RS).

\begin{tabular}{|c|c|c|c|c|c|c|}
\hline & RBI & PET & PER & PEE & Totais $\mathrm{sp} / \mathrm{nc}$ & Total RS \\
\hline Acontiinae & 5 & 7 & 2 & 5 & $8 / 4$ & 16 \\
\hline Acronictinae & 0 & 0 & 1 & 1 & $1 / 0$ & 2 \\
\hline Agaristinae & 0 & 1 & 0 & 1 & $2 / 0$ & 5 \\
\hline Amphipyrinae & 24 & 52 & 43 & 61 & $57 / 27$ & 84 \\
\hline Bagisarinae & 0 & 1 & 1 & 1 & $1 / 0$ & 2 \\
\hline Catocalinae & 3 & 15 & 9 & 6 & $14 / 4$ & 28 \\
\hline Cuculliinae & 1 & 0 & 0 & 1 & $1 / 0$ & 3 \\
\hline Euteliinae & 1 & 0 & 2 & 2 & $2 / 0$ & 4 \\
\hline Hadeninae & 14 & 16 & 18 & 24 & $26 / 9$ & 47 \\
\hline Heliothinae & 3 & 2 & 3 & 4 & $6 / 2$ & 7 \\
\hline Herminiinae & 3 & 4 & 2 & 3 & $1 / 3$ & 5 \\
\hline Noctuinae & 11 & 3 & 9 & 6 & $16 / 5$ & 29 \\
\hline Nolinae & 1 & 0 & 0 & 0 & $0 / 1$ & 0 \\
\hline Ophiderinae & 9 & 32 & 20 & 15 & $36 / 11$ & 103 \\
\hline Pantheinae & 0 & 0 & 0 & 1 & $1 / 0$ & 1 \\
\hline Plusiinae & 4 & 2 & 3 & 2 & $6 / 0$ & 13 \\
\hline Sarrothripinae & 4 & 0 & 1 & 2 & $2 / 3$ & 4 \\
\hline Stictopterinae & 0 & 0 & 0 & 0 & $0 / 0$ & 1 \\
\hline Total & 83 & 134 & 114 & 135 & $180 / 69$ & 354 \\
\hline
\end{tabular}

de indivíduos por espécie de todos os locais $(23,97)$ evidenciando a forte pressão de uso agrícola no seu entorno, maximizada pela sua pequena área. Neste local as três espécies mais abundantes relacionam-se com as plantas cultivadas nas proximidades, especialmente soja. Estas observações concordam com o fato de que os efeitos da perturbação ambiental dependem do tamanho das áreas a serem preservadas (Hamer \& Hill 2000). Ilustrando isto, $A$. gemmatalis, conhecida como lagarta-da-soja, foi a espécie mais abundante com 1191 indivíduos seguida por Condica sutor (Guenée, 1852) (249) que é hóspede de diversas plantas invasoras e de aipo (Umbeliferae) (Crumb 1956) e Spodoptera cosmioides (Walker, 1858) (157) que é hóspede de diversas culturas de interesse econômico (Silva et al. 1968). Apesar deste parque fazer parte do Bioma Mata Attântica (Brasil 2000) a riqueza de espécies de Amphipyrinae e Ophiderinae (Tabela II) foi relativamente pequena.

No parque Estadual do Espigão Alto foram coletadas, à semelhança do Parque Estadual Florestal do Turvo, 135 espécies, porém observou-se a predominância de Amphipyrinae e Hadeninae (Tabela II). O número de espécies representantes de Ophiderinae representou menos da metade do observado no Parque Estadual do Turvo (Tabela II). Neste Parque foram coletadas 31 espécies únicas, o que demonstra a sua importância para preservação. Este parque, de tamanho reduzido (1.331,9 ha), também situa-se em uma área de extrema importância biológica das áreas prioritárias para a conservação da biodiversidade da Mata Atlântica (Brasil 2000). Provavelmente o grande número de espécies observado esteja relacionado com o seu excelente estado de conservação e por estar conectado com diversas áreas de mata preservada que se continuam pelo vale do Rio Pelotas. Observou-se também, em função da altitude, a ocorrência de espécies típicas do Bioma Campos Sulinos, de Cima da Serra, como Anicla temperata (Schaus, 1894) e Tripseuxoa deeringi Schaus, 1929 (Specht 2001).

Apesar dos ecossistemas pertencentes aos Campos Sulinos apresentarem riqueza menor que os pertencentes ao Bioma Mata Atlântica (Brasil 2000), há necessidade da manutenção de Unidades de Conservação em ambos os Biomas, tanto pelo número de espécies abrigadas quanto pela falta de conhecimento sobre elas.

Este estudo, a exemplo de outros (Barlow \& Woiwod 1989; Holloway \& Barlow 1992; Marinoni \& Dutra 1996; Ferreira et al. 1995; Lübeck et al. 1995; Chey et al. 1997; Intachat et al. 1997; Marinoni et al. 1997; Woiwod 1997; Usher \& Keiller 1998; Camargo 1999; Kitching et al. 2000; Specht 2001; Specht \& Corseuil 2002b; Teston \& Corseuil 2004) demonstrou a possibilidade da utilização de grupos de lepidópteros para obter informações ecológicas sobre áreas de preservação, em tempo relativamente curto e custos muito pequenos (Oliver \& Beattie 1996). Além disto, devido à incorporação dos exemplares em coleções de referência, seus dados permitem ampliar a distribuição geográfica e inventariamento das espécies contribuindo para o conhecimento da biodiversidade local, regional e mais amplamente global (Kremen et al. 1993).

Os maiores valores de riqueza observados em Amphipyrinae, Ophiderinae e Hadeninae concordam com os 
Tabela III - Número de espécies de noctuídeos, exclusivas e/ou de ocorrência simultânea, capturados com armadilha luminosa em quatro Áreas Estaduais de Conservação do Rio Grande do Sul. (RBI - Reserva Biológica do Ibirapuitã, PET - Parque Estadual do Turvo, PER - Parque Estadual de Rondinha e PEE - Parque Estadual do Espigão Alto).

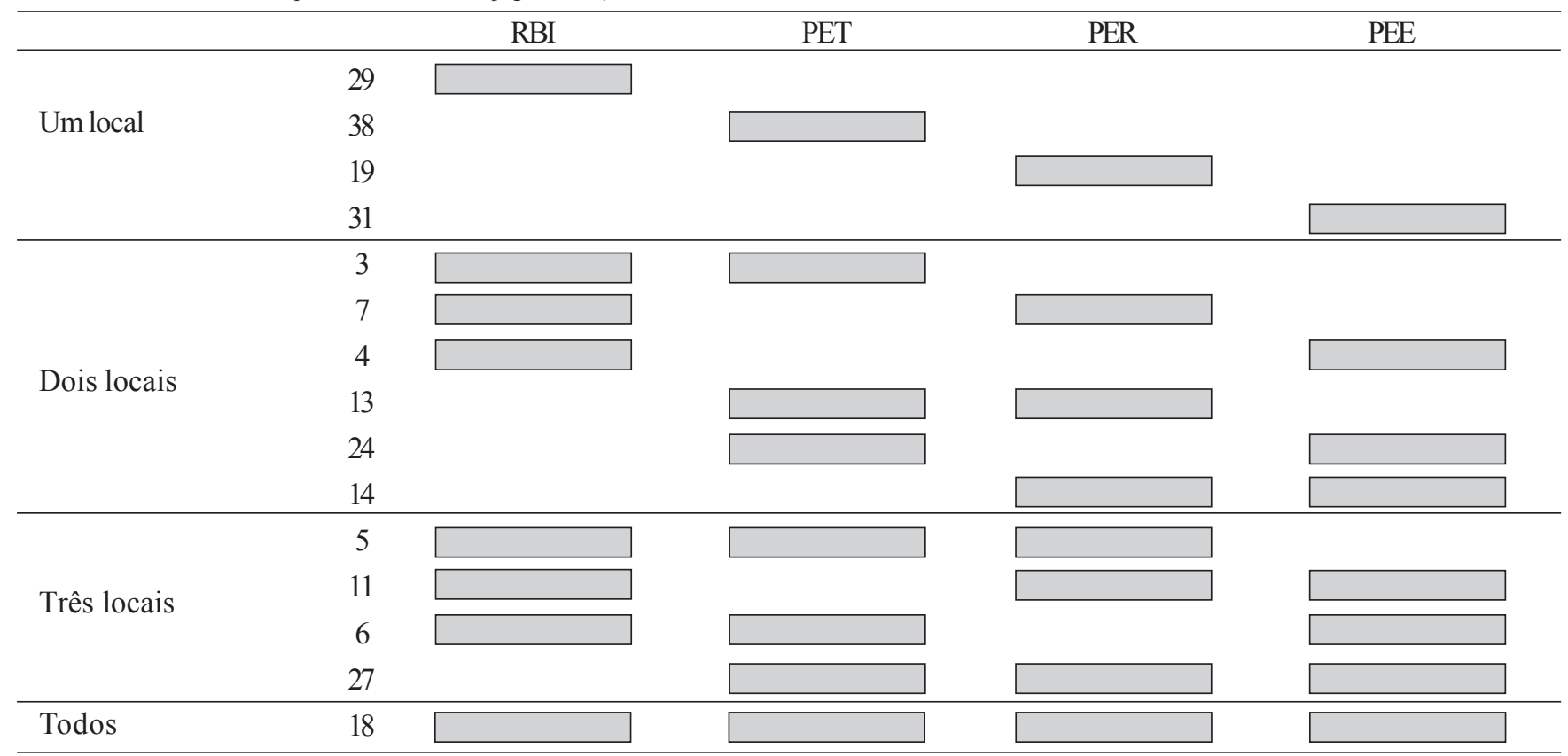

referidos para Santa Maria, RS (Link 1977) e Salvador do Sul, RS (Specht \& Corseuil 2002b). Observa-se que, como destacado pelos últimos autores, Ophiderinae foi representada por número de indivíduos relativamente pequeno, especialmente quando se exclui $A$. gemmatalis, que é praga da cultura da soja. Como demonstrado para a comunidade de Noctuidae de Salvador do Sul, RS (Specht \& Corseuil 2002b) e Santa Maria, RS (Link 1977), as espécies que apresentaram as maiores abundâncias são espécies-praga de determinadas culturas ou relacionam-se a plantas invasoras ou muito comuns. Assim como neste estudo, a variação específica entre localidades em função da vegetação predominante também é descrita para cultivos e, especialmente, florestas (Lin 1995; Chey et al. 1997; Intachat et al. 1997; Usher \& Keiller 1998).

Apesar dos conhecimentos sobre os noctuídeos neotropicais serem relativamente escassos, a avaliação das características próprias de cada comunidade, neste estudo através da riqueza e abundância permitiram caracterizar as comunidades de cada uma das unidades de conservação. Devido às amostragens terem sido efetuadas em períodos muito curtos, as informações, mesmo que de forma limitada, contribuíram para a ampliação do inventariamento e distribuição geográfica das espécies no Estado. Considerando que vários grupos de mariposas, incluindo Noctuidae, são indicadores de qualidade ambiental (Kitching et al. 2000), recomenda-se que sejam efetuados levantamentos nas diferentes épocas e em vários anos (Specht 2001) pois com o conhecimento de toda a comunidade e suas características ao longo do ano, estes lepidópteros poderão ser utilizados para avaliar as mudanças ambientais e seus agentes causais.

$\mathrm{O}$ presente estudo permitiu constatar que o número de noctuídeos ocorrentes no Rio Grande do Sul é bem maior que as 354 espécies já referidas e que as Unidades Estaduais de Conservação constituem importantes meios para ampliação dos conhecimentos sobre este grupo e para a manutenção da sua diversidade .

Agradecimentos. Ao Engenheiro Agrônomo Alexandre Carvalho da Empresa Magna Engenharia, pela operacionalização e viabilização dos trabalhos de campo.

\section{REFERÊNCIAS}

Arend, L. M. 1997. Geografia Física, p. 22-53. In: G. R. Hoffmann; L. M. Arend; J. C. B. Silveira; H. R. Bellomo (org.) \& J. L. M. Nunes. Rio Grande do Sul-aspectos da Geografia. $4^{\mathrm{a}}$ ed. Porto Alegre, Martins Livreiro, $104 \mathrm{p}$.

Barlow, H. S. \& I. P. Woiwod. 1989. Moth diversity of a tropical forest in Peninsular Malaysia. Journal of Tropical Ecology 5: 37-50.

Brasil. 2000. Avaliação e ações prioritárias para a conservação da biodiversidade da Mata Atlântica e Campos Sulinos. Brasília, Ministério do Meio Ambiente / Secretaria de Biodiversidade e Florestas. 40 p.

Camargo, A. J. A. 1999. Estudo comparativo sobre a composição e a diversidade de lepidópteros noturnos em cinco áreas da Região dos Cerrados. Revista Brasileira de Zoologia 16: 369-380.

Chey, V. K.; J. D. Holloway \& M. R. Speight. 1997. Diversity of moths in forest plantations and natural forests in Sabah. Bulletin of Entomological Research 87: 371-385.

Crumb, S. E. 1956. The larvae of the Phalaenidae. Washington, U.S. Government Printing Office, 356 p. [United States Department of Agriculture Technical Bulletin $\left.n^{\circ} 1135\right]$.

Ferreira, P. S. F.; A. S. Paula \& D. S. Martins. 1995. Análise faunística de Lepidoptera Arctiidae em área de reserva natural remanescente de floresta tropical em Viçosa, Minas Gerais. Anais da Sociedade Entomológica do Brasil 24: 123-133. 
Frost, S. W. 1957. The Pennsylvania Insect Light Trap. Journal of Economic Entomology 50: 287-292.

Fry, R. \& P. Waring. 1996. A guide to moth traps and their use. The Amateur Entomologist 24: 1-60.

Gardiner, B. O. C. 1995. The very first light-trap, 1565? Entomologist Record 107: 45-46.

Gaston, K. J. 1988. Patterns in the local and regional dynamics of moths populations. Oikos 53: 49-57.

Hamer, K. C. \& J. K. Hill. 2000. Scale-dependent effects of habitat disturbance on species richness in tropical forests. Conservation Biology 14: 1435-1440.

Hampson, G. F. 1903-1913. Catalogue of the Lepidoptera Phalaenae in the Britihs Museum. London, Taylor and Francis, V. 4-13. Pranchas 55-239.

Heppner, J. B. 1991. Faunal regions and the diversity of Lepidoptera. Tropical Lepidoptera 2: 1-85.

Holloway, J. D. \& H. S. Barlow. 1992. Potential for loss of biodiversity in Malaysia, illustrated by the moth fauna, p. 293-311. In: A. Aziz; S. A. Kadir \& H. S. Barlow (Eds.). Pest management and the environment in 2000. Wallingford, $C A B$ International \& Agricultural Institute of Malaysia, $401 \mathrm{p}$.

Holloway, J. D.; J. D. Bradley \& D. J. Carter. 1992. II E Guides to Insects of Importance to Man. 1. Lepidoptera. London, The Natural History Museum, $263 \mathrm{p}$.

Intachat, J.; J. D. Holloway \& M. R. Speight. 1997. The effects of different forest management practices on geometrid moth populations and their diversity in peninsular Malaysia. Journal of Tropical Forest Science 9: 411-430.

Kitching, R. L.; A. G. Orr; L. Thalib; H. Mitchell; M. S. Hopkins \& A. W. Graham. 2000. Moth assemblages as indicators of environmental quality in remnants of upland Australian rain forest. Journal of Applied Ecology 37: 284-297.

Kremen, C.; R. K. Colwell; T. L. Erwin; D. D. Murphy; R. F. Noss \& M. A. Sanjayan. 1993. Terrestrial arthropod assemblages: Their use in conservation planning. Conservation Biology 7: 796-808.

Lafontaine, J. D. \& R. W. Poole. 1991. Noctuoidea: Noctuidae (part.): Plusiinae. Washington, The Wedge Entomological Research Foundation, 182 p. [Série: The moths of America North of Mexico, including Greenland; fasc. 25.1].

Lin, C. S. 1995. Macroheteroceran diversity variation in agricultural/ forestry systems at Meifeng. Journal of Taiwan Museum 48: 25-38.

Link, D. 1977. Abundância relativa de alguns Noctuidae, em armadilha luminosa, em Santa Maria, RS. Revista do Centro de Ciências Rurais 7: 331-351.

Lübeck, G. M.; J. V. Oliveira \& R. P. Almeida. 1995. Análise faunística de lepidópteros coletados em duas comunidades agrícolas da Zona da Mata Norte de Pernambuco. Anais da Sociedade Entomológica do Brasil 42: 353-370.

Marinoni, R. C. \& R. R. C. Dutra. 1996. Levantamento da fauna entomológica do Estado do Paraná. II. Ctenuchidae (Lepidoptera). Revista Brasileira de Zoologia 13: 435-461.

Marinoni, R. C.; R. C. C. Dutra \& M. M. Casagrande. 1997. Levantamento da fauna entomológica do Estado do Paraná. III. Saturniidae (Lepidoptera). Revista Brasileira de Zoologia 14: 473-495.
Muirhead-Thompson, R. C. 1991. Trap responses of flying insects. The influence of trap design on capture efficiency. London, Academic Press, 287 p.

Oliver, I. \& A. J. Beattie. 1996. Designing a cost-effective invertebrate survey: a test of methods for rapid assessment of biodiversity. Ecological Applications 6: 594-607.

Poole, R. W. 1989. Noctuidae. In: J. B. Heppner (ed.) Lepidopterorum Catalogus. New York, Brill, 1314 p. (3v.).

Poole, R. W.; C. Mitter \& M. Huettel. 1993. A revision and cladistic analysis of the Heliothis virescens species-group (Lepidoptera: Noctuidae) with a preliminary morphometric analysis of Heliothis virescens. Mississippi Agricultural and Forestry Experiment Station Technical Bulletin 185: 1-51. [Mississippi Entomological Museum n ${ }^{\circ}$ ]

Scoble, M. J. 1992. The Lepidoptera Form, Function and Diversity. New York, Oxford University Press, $404 \mathrm{p}$.

Seitz, A. 1919-1944. Die Gross-Schmeterlinge der Erde. Abteilung II - Die Gross-Scheterlinge des Amerikanischen Faunengebietes. Band 7: Eulenartige Nachtfalter. Stuttgart, Alfred Kernen, 508 p., 96 pranchas.

Sema - Secretaria do Meio Ambiente do Estado do Rio Grande do Sul. Unidades de Conservação. Porto Alegre: PROCERGS. Copyright 2002. Publicado em http://www.sema.rs.gov.br/sema/html/ bioconh1.htm.

Silva, A. G. de A.; C. R. Gonçalves; D. M. Galvão; A. J. L. Gonçalves; J. Gomes; M. M. Silva \& L. Simoni. (1968). Quarto Catálogo dos insetos que vivem nas plantas do Brasil: seus parasitos e predadores. Tomo 1, parte 2 Insetos, Hospedeiros e inimigos naturais. Rio de Janeiro, Ministério da Agricultura, $622 \mathrm{p}$.

Specht, A. 2001. Diversidade e aspectos ecológicos dos noctuídeos (Lepidoptera, Noctuidae) ocorrentes no Rio Grande do Sul, Brasil. Tese de Doutorado. Porto Alegre, Pontifícia Universidade Católica do Rio Grande do Sul, 71 p.

Specht, A. \& E. Corseuil. 1996. Lista documentada dos noctuídeos (Lepidoptera: Noctuidae) ocorrentes no Rio Grande do Sul, Brasil. Biociências 4: 131-170.

Specht, A. \& E. Corseuil. 1998. Novas ocorrências de noctuídeos (Lepidoptera, Noctuidae) no Rio Grande do Sul, Brasil. Biociências 6: 123-129.

Specht, A. \& E. Corseuil. 2001. Ocorrência de noctuídeos, (Lepidoptera, Noctuidae) no Rio Grande do Sul, Brasil. Nota suplementar I. Biociências 9: 97-103.

Specht, A. \& E. Corseuil. 2002a. Ocorrência de noctuídeos, (Lepidoptera, Noctuidae) no Rio Grande do Sul, Brasil. Nota suplementar II. Biociências 10: 169-74.

Specht, A. \& E. Corseuil. 2002b. Diversidade dos noctuídeos (Lepidoptera, Noctuidae) em Salvador do Sul, RS, Brasil. Revista Brasileira de Zoologia 19: 281-298.

Teston, J. A.; Corseuil, E. 2004. Diversidade de Arctiinae (Lepidoptera, Artiidae) capturados com armadilha luminosa, em seis comunidades do Rio Grande do Sul, Brasil. Revista Brasileira de Entomologia 48: $77-90$

Usher, M. B. \& S. W. J. Keiller. 1998. The macrolepidoptera of farm woodlands: determinants of diversity and community structure. Biodiversity and Conservation 7: 725-748.

Woiwod, I. P. 1997. Detecting the effects of climate change on Lepidoptera. Journal of Insect Conservation 1: 149-158.

Recebido em 14.V.2004; aceito em 12.XI.2004 